\title{
The prevalence of co-morbidities in patients on HAART referred for physiotherapy in three public hospitals in KwaZulu-Natal, South Africa: A short report
}

\begin{abstract}
Introduction: The roll-out of Highly Active Antiretroviral Therapy (HAART) to patients infected with the Human Immuno-deficient Virus (HIV) is presenting with co-morbidities as a result of the side effects of the drugs or the prolonged lifespan of the HIV positive individual. This poses a challenge to physiotherapists for rehabilitation which has changed from prophylaxis and management of opportunistic infections to managing the co-morbidities. The aim of this preliminary study was to determine the number of patients on HAART with co-morbidities admitted to three public hospitals in KwaZulu-Natal and referred for physiotherapy.
\end{abstract}

\section{Maharaj SS, D.Phil' Rangiah P, MPhysT ${ }^{1}$}

1 University of KwaZulu-Natal.

Method: This descriptive epidemiological study used a cross-sectional design to determine the prevalence of co-morbidities associated with HAART. Baseline data of patients and their demographics, AIDS status and clinical co-morbidities were recorded. The sample consisted of 78 patients with an average CD4 count of 338 cells $/ \mathrm{mm}^{3}$ and a viral load of 133 copies $/ m L$. Patients were categorised according to their HIV stages: 13 (17\%) asymptomatic, 44 (56\%) 'AIDS-related complex' and 21 (27\%) had full blown AIDS.

Results: Patients referred for physiotherapy presented with peripheral neuropathy $36(46 \%)$, pulmonary tuberculosis $29(37 \%)$, musculo-skeletal pain 17(22\%), myopathies $9(12 \%)$, pneumonia $8(10 \%)$, enthesopathies $4(5 \%)$, cerebral vascular accidents $2(3 \%)$ and abdominal pain $1(1 \%)$.

Conclusion: To rehabilitate the HIV infected individual successfully physiotherapists should note the side effects and co-morbidities associated with HAART.

\section{KEY WORDS: HIV, PROFILE, ADMISSION, REFERRAL, PHYSIOTHERAPY.}

\section{INTRODUCTION}

Highly Active Antiretroviral Treatment (HAART), a triple combination of anti-retroviral drugs (ARVs), has been provided in South Africa since April 2004. Patients with a CD4 count of less than 200 cells $/ \mathrm{mm}^{3}$ are eligible for HAART which is prolonging their survival (Wong et al, 2004) with an increase in chronic health problems, impairments and disabilities compared to the pre-HAART era (Rusch et al, 2003). The infected individual is in transition between an acute terminal and a sub-acute chronic disease (Brown et al, 2000). Patient care will change from prophylaxis and the management of opportunistic infections to managing co-morbidities associated with HAART which will continue to increase beyond 2015 (Dorrington et al, 2004).

Health care professionals, social workers and religious leaders enable the HIV infected individuals to have a longer life span (Addington-Hall \&
Higginson 2001) with co-morbidities associated with the side effects of antiretroviral drugs often requiring rehabilitation. Physiotherapists will have to manage HIV related syndromes and co-morbidities affecting the respiratory, neurological and musculoskeletal systems. Physiotherapy is shown to have beneficial effects on pain reduction and improving the health-related quality of life in an HIV affected person (Germaine et al, 2005). To date, Medline, Pubmed, Pedro, Biomed and a wide Google search showed no published studies of co-morbidities and the number of patients on HAART referred for physiotherapy in South Africa. The purpose of this preliminary study was therefore to:

1. determine the profile and number of in-patients on HAART referred for physiotherapy

2. rank the associated co-morbidities.

This data will have clinical relevance for the changing profile and increasing workload of HIV/AIDS patients and the physiotherapy profession.

\section{METHODOLOGY}

\section{Research Design}

This descriptive epidemiological study used a cross-sectional design to determine the prevalence of co-morbidities associated with HAART during the period June 2007 to July 2008. The ethics committee of the University of KwaZulu-Natal approved this study (FECHS036/07). Informed consent was obtained from the patients prior to participation in this study.

Correspondence to:

Dr. S. S. Maharaj

Head: Dept. of Physiotherapy

University of KwaZulu-Natal

Westville Campus

KwaZulu-Natal, Durban.

E-mail: maharajss@ukzn.ac.za 


\section{Research setting}

Three public hospitals servicing a large proportion of the urban and semiurban population of Durban viz. King Edward VIII, Prince Mshiyeni and R.K. Khans were identified. Each hospital had an antiretroviral clinic and an efficient cross-referral system. HAART was a combination of at least three antiretroviral drugs that included either a protease inhibitor or a non-nucleoside reverse transcriptase inhibitor as defined by Brown et al (2006).

\section{Sample}

The population consisted of HIV/AIDS patients referred for admission. The sample was based on the following.

Inclusion criteria:

- patients on a HAART regime

- medically diagnosed co-morbidities

- $\quad$ referred for physiotherapy

\section{Exclusion criteria:}

- default of HAART regime

- incomplete medical records

- non-compliance of medical information with the researchers

\section{Procedure}

HAART patients admitted to the respective hospitals for physiotherapy were identified by the physiotherapists in charge who notified the researchers. Patients were predominantly from the medical, surgical and orthopaedic wards and the researchers recorded the relevant data for patients satisfying the inclusion criteria from the in-patient files. This included: age and gender, specific co-morbidity, CD4 count and viral loads. The HIV stages of the patients were classified according to the Centres for Disease Control and Prevention (1992). These were asymptomatic stage (with few outward signs and symptoms), AIDS related complex (the development of one or more opportunistic infections) and AIDS (serious opportunistic infections and cancer). Confusing or missing information in the in-patients file was clarified or sourced from the referring medical practitioner, physiotherapist in-charge of the unit or the patient.

\section{Statistical analysis}

The data was analysed using Microsoft Excel and SPSS version 15 and summarised using descriptive statistics. The co-morbidities were classified into eight common conditions. The most common was ranked 1 and the least common 8 (table 3). To ensure internal validity regarding sample selection bias only patients referred from the ARV sites who had not defaulted on their antiretroviral regime were selected. Information bias was minimized by transcribing information directly and accurately from the in-patient's file. Patients having two or more co-morbidities were analysed individually for statistical purposes.

\section{RESULTS}

A total of 138 patients on HAART were admitted during the study period. From these $60 \% \quad(n=83)$ were referred for physiotherapy with $94 \%(n=78)$ satisfying the inclusion criteria. Five patients were excluded because three files lacked statistical information and two were non-compliant with verbal communication. Table 1 indicates the demo-

Table 1: Demographic profile of sample $(n=78)$

\begin{tabular}{|l|l|}
\hline Patients' characteristics & Frequencies and proportions (N) \\
\hline Gender & $32(41)$ \\
Female (41\%) & $46(59)$ \\
Male (59\%) & \\
\hline Average age (years) & $30.9( \pm 5.1)$ \\
Female (SD) & $34.7( \pm 6.3)$ \\
Male (SD) & $\underline{\text { Min -Max }}$ \\
\hline Age range (years) & $23-41$ (years) \\
Female & $19-52$ (years) \\
Male &
\end{tabular}

Table 2: Category of HIV profile of sample $(n=78)$

\begin{tabular}{|c|c|}
\hline HIV stages & Sample N(\%) \\
\hline $\begin{array}{l}\text { Asymptomatic HIV (\%) } \\
\text { AIDS-related complex (\%) } \\
\text { AIDS (\%) }\end{array}$ & $\begin{array}{l}13(17) \\
44(56) \\
21(27)\end{array}$ \\
\hline $\begin{array}{l}\text { Average CD4 (SD) } \\
\quad \text { Range }\end{array}$ & $\begin{array}{l}338 \mathrm{cell} s / \mathrm{mm}^{3}( \pm 282) \\
179-401 \mathrm{cells} / \mathrm{mm}^{3}\end{array}$ \\
\hline $\begin{array}{l}\text { Average viral load (SD) } \\
\quad \text { Range }\end{array}$ & $\begin{array}{l}132.69 \text { copies } / \mathrm{mL}( \pm 309) \\
108-368 \text { copies } / \mathrm{mL}\end{array}$ \\
\hline
\end{tabular}

graphic characteristics of the sample and Table 2 the HIV profile. The average CD4 count was 338 cells $/ \mathrm{mm}^{3}( \pm 282)$ and viral load 132.69 copies $/ \mathrm{mL}( \pm 309)$. Category of the HIV stages of the patients were $17 \%(n=13)$ asymptomatic, $56 \%$ $(\mathrm{n}=44)$ AIDS related complex and $27 \%$ $(n=21)$ had full blown AIDS. Table 3 illustrates the co-morbidities and their ranking. From the patients presenting with musculo-skeletal pain ( $\mathrm{n}=17)$, seven had upper limb and ten lower limb pain; myopathies $(n=9)$ had three upper and six lower limb involvement; enthesopathies $(n=4)$, were restricted to the lower limbs with three having Achilles tendon and one ilio-tibial pain.

\section{DISCUSSION}

This study was designed to determine the co-morbidities of patients receiving HAART and referred for physiotherapy. A study by Myezwa et al (2007), in the pre-HAART era, reported that $19 \%$ $(n=139)$ of the possible 344 HIV patients were considered for physiotherapy of which $2 \%(n=3)$ were referred. The respiratory system was the most affected .

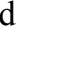


with pneumonia manifesting in $29 \%$ followed by meningitis $25 \%$ and gender male. In our study from a possible 138 patients on HAART, $60 \%(n=83)$ were referred for physiotherapy. The ranking of the co-morbidities was $46 \%$ peripheral neuropathy, 37\% tuberculosis (TB) and $10 \%$ pneumonia with $41 \%$ female and $59 \%$ male. A possible explanation for our findings is that HAART is "changing the profile" of the HIV infected individuals. Studies have documented a link between the central or peripheral nervous system, peripheral neuropathy and the HIV disease which is associated with antiretroviral therapy (Parry et al, 1997; Moyle \& Sadler 1998). Our study supports this as $46 \%(n=36)$ presented with peripheral neuropathy and $3 \%(n=2)$ cerebral vascular accident. Our data show the lower limbs were more affected $(n=19)$ compared to the upper limbs $(n=8)$ and a combination of upper and lower limbs $(n=9)$. Although the specific pathophysiology is not understood (Moyle \& Sadler 1998) noted that several nucleoside antiretroviral agents such as zalcitabine, stavudine and didanosine lead to patients developing peripheral neuropathy within three months. This antiretroviral implication for peripheral neuropathy was supported by Gerschenson and Brinkman (2004) attributing the HIV nucleoside reverse transcriptase inhibitors as a possible composition was $57 \%$ female and $43 \%$

cause of mitochondrial injury to the host cells.

HIV patients co-infected with TB receiving ARV therapy sometimes have a paradoxical worsening of symptoms. This is due to reconstitution of the immune response to mycobacteria by Rifampicin, an anti-TB drug, which metabolises protease inhibitors and non-nucleoside reverse transcriptase inhibitors (McShane 2005). This study did not identify the prevalence of TB before the administration of HAART and the authors assumed their sample probably had TB prior to the commencement of antiretroviral therapy supported by Whalen et al (1995) where co-infection with HIV and mycobacterium tuberculosis results in TB being the commonest co-infection in HIV individuals.

Myezwa et al (2007) ranked pneumonia $(29 \%)$ the most common diagnosis compared to $10 \%$ of our sample could be related to the mean CD4 cell count of 338 cells $/ \mathrm{mm}^{3}$ for our patients. The AIDS Surveillance Data Project found patients with a CD4 count of less than $200 \mathrm{cell} / \mathrm{mm}^{3}$, as in the pre-HAART era, had a five times greater chance of acquiring bacterial pneumonia compared to a higher CD4 cell count (CDC, 1992).

Musculo-skeletal pain, myopathies and enthesopathies data from this study are relevant as Gerschenson and Brinkman (2004) report that long term exposure to

Table 3: Co-morbidities referred for Physiotherapy $(n=78)$

\begin{tabular}{|l|l|l|}
\hline Rank & Co-morbidities & Frequency N (\%) \\
\hline 1 & Peripheral neuropathy & $36(46)$ \\
\hline 2 & TB & $29(37)$ \\
\hline 3 & Musculo-skeletal pain & $17(22)$ \\
\hline 4 & Myopathies & $9(12)$ \\
\hline 5 & Pneumonia & $8(10)$ \\
\hline 6 & Enthesopathies & $4(5)$ \\
\hline 7 & CVA & $2(3)$ \\
\hline 8 & Abdominal pain & $1(1)$ \\
\hline
\end{tabular}

'The total exceeds $100 \%$ because some patients had two or more co-morbidities antiretroviral therapy and HIV causes mithochondrial toxicities which could lead to myopathies, pain and enthesopathies. Incidence of co-morbid conditions could result in pain (Simpson \& Tagliati, 1994) as noted in this study.

The data indicate that enthesopathies was restricted to the lower limbs with three patients having the Achilles tendon and one ilio-tibial pain. Musculo-skeletal pain and myopathies were more common in the lower limbs. It is not clear whether this greater prevalence of lower limb involvement was due to HAART medication or to the functional stress of weight bearing.

One female patient was referred for abdominal pain because females with pelvic inflammatory pain are requested to be managed by shortwave diathermy. The patient's file however did not confirm the presence or absence of pelvic inflammatory disease.

\section{CONCLUSION}

Since no published studies described the co-morbidities of patients on HAART referred for physiotherapy in South Africa, this study was an appropriate starting point. The results compared to a similar study in the pre-HAART era, shows that HAART is changing the profile of the HIV individual. The data indicate that physiotherapy referrals are changing from acute respiratory co-morbidities to peripheral neuropathy, myopathies, enthesopathies and musculo-skeletal pain. The lower limbs are more affected compared to upper limbs. As a preliminary study from three public hospitals further research is needed to establish what co-morbidities and functional deficits exist for the physiotherapy profession in the HAART-era.

\section{LIMITATION OF THE STUDY}

The researchers acknowledge that the study was limited to in-patients with referrals based on the medical practitioner's discretion. As data collectors the researchers could have been bias although case notes were meticulously examined and information transcribed exactly from the patient's files. The authors accept that superficial data analysis and generalisation of the findings can be explored with further research. 


\section{ACKNOWLEDGEMENTS}

The authors thank the physiotherapists, medical practitioners and nursing staff from the public hospitals for assisting with the study.

\section{REFERENCES}

Addington-Hall MJ, Higginson JL. 2001. Palliative care for non-cancer patients. London: Oxford Press.

Brown DM, Thorne JE, Foster GL, Duncan JL. 2006. Factors affecting attrition in a longitudinal study of patients with AIDS. AIDS Care 18(7): 821-829.

Brown L, Lourie K, Pao M. 2000. A review of children and adolescents living with HIV and AIDS. Journal of Child Psychology and Psychiatry 41(1): 81-96.

Centres for Disease Control and Prevention. 1992. 1993 revised classification system for HIV infection and expanded surveillance case definition for AIDS among adolescents and adults. Morbidity and Mortality Weekly Report 41(17): 1-19.
Dorrington R, Bradshaw D, Johnon L, Budlender D. 2004. The demographic impact of HIV/ AIDS in South Africa. Cape Town: Centre for Actuarial Research and South African Medical Research Council.

Germaine A, Griwold ES, Spielman L, Fishman B. 2005. Coping strategies of HIV patients with peripheral neuropathy. AIDS Care 17(6): 711-720.

Gerschenson M, Brinkman K. 2004. Mithochondrial dysfunction in AIDS and its treatment. Mitochondrion 4: 763-777.

McShane H. 2005. Co-infection with HIV and TB: double trouble. International Journal of Sexually Transmitted Disease and AIDS 16: 95-101.

Moyle GJ, Sadler M. 1998. Peripheral neuropathy with nucleoside antiretroviral therapy: risk factors, incidence and management. Drug Safety 19: 481-494.

Myezwa H, Stewart A, Mbambo N, Nesara P. 2007. Status of referral to physiotherapy among HIV positive patients at Chris Hani Baragwanath Hospital Johannesburg South Africa. 2005. South African Journal of Physiotherapy 63(2): 27-31.
Parry O, Mielke J, Latif AS, Ray S, Levy LF, Siziya S. 1997. Peripheral neuropathy in individuals with HIV infections in Zimbabwe. Acta Neuologica Scandinavica 96: 218-222.

Rusch M, Nixon S, Schilder A, Braitstein P, Chan K, Hogg B. 2003. Disability among people living with HIV in British Columbia: the unacknowledged epidemic. Canadian Journal of Infectious Disease 14: 17.

Simpson DM, Tagliati M. 1994. Neurological manifestations of HIV infection. Annals of Internal Medicine 121: 769-785.

Whalen C, Horsburgh CR, Hom D, Lahart C, Simberkoff M, Ellner J. 1995. Accelerated course of human immunodeficiency virus infection after tuberculosis. American Journal of Respiratory Critical Care Medicine 151: 129-135.

Wong KH, Chan KC, Lee SS. 2004. Delayed progression to death and to AIDS in a Hong Kong cohort of patients with advanced type-1 disease during the era of highly active antiretroviral therapy. Clinical Infectious Diseases 39: 853-860. 